\title{
Compressible Hot Fluid Injection into an Isothermal Pervious/Adiabatic Impervious Half Space
}

\author{
John C.-C. Lu ${ }^{1, *}$ and Feng-Tsai Lin $^{2}$ \\ ${ }^{1}$ Department of Civil Engineering, Chung Hua University, No. 707, Sec. 2, Wufu Rd., Hsinchu 30012, Taiwan R.O.C. \\ ${ }^{2}$ Department of Naval Architecture and Ocean Engineering, National Kaohsiung University of Science and Technology, No. 142, \\ Haijhuan Rd., Kaohsiung 81157, Taiwan R.O.C. \\ ${ }^{*}$ Corresponding author
}

\begin{abstract}
The study is concentrated on the isotropic elastic responses of a homogeneous isothermal pervious surface or adiabatic impervious surface due to injection of compressible hot fluid. Based on the theory of thermal elasticity of porous media, the analytical models are developed for the ground deformation with excess pore fluid pressure and temperature increment of the strata due to thermal fluid influences. Using Hankel transform to present the closed-form solutions of the analytical model, the investigation can improve understanding of the isotropic porous strata due to injection of compressible hot fluid.
\end{abstract}

Keywords-hot fluid injection; half space; Hankel transform; closed-form solution

\section{INTRODUCTION}

Strata responses due to hot fluid injection is an important engineering issue. Considering impact on engineering safety, many studies were concentrated on mechanical, thermal and hydraulic behavior due to thermal consolidation. Hydraulic and thermal disturbance in the strata usually result in a volumetric or temperature change of solid skeleton and fluid. The change can increase excess pore fluid pressure and lead to a decrease in effective stress. The shear resistance loss of solid skeleton may result in a hydraulic or thermal failure in the strata. The simulation and its validation is a major concern for the improvement of engineering safety.

An analytical model for the areal distribution of land deformation due to hot water injection of a confined and leaky aquifers was developed by Bear and Corapcioglu [1]. Compared to conventional water flooding in recovering heavy oil from heterogeneous reservoirs, Alajmi et al. [2] presented the performance of hot water flooding through fine-mesh numerical simulations. In the investigation of using dual horizontal wells by Sasaki et al. [3], a system of gas production from methane hydrate layers involving hot water injection was investigated. Rosenbrand et al. [4] addressed permeability change in sandstone due to heating from $20^{\circ} \mathrm{C}$ to $70 \sim 200^{\circ} \mathrm{C}$. Transient ground surface displacements produced by a point heat source or sink through analog quantities between poroelasticity and thermoelasticity were displayed by $\mathrm{Lu}$ and Lin [5]. To simulate gas production from methane hydrate-bearing sand by hot water cyclic injection, a three-dimensional middle size reactor was used by Yang et al. [6].
On the basis of thermally elasticity of porous media, the present investigation is focused on the closed-form solutions of an isothermal pervious or adiabatic impervious ground space due to injection of compressible hot fluid which still have not been derived in previous studies. The soil or rock mass is modelled as a linearly elastic medium with isotropic properties in this paper. The mechanical properties, thermal conductivity and hydraulic fluid flow are treated as isotropic. Using Hankel integral transform, the closed-form solutions of temperature increment, excess pore fluid pressure and displacements of the strata due to hot fluid injection are obtained. The results can improve understanding of the compressible thermally fluid injection induced responses.

\section{MATHEMATICAL MODEL}

\section{A. Governing Equations}

Figure 1 displays an isothermal pervious or adiabatic impervious porous elastic strata subjected to a point hot fluid injection. The soil or rock is modeled as a homogeneous isotropic media. The mechanical governing equations are derived by equilibrium equations, constitutive law and kinematics relationships in equations (1a) and (1b). The injection point of compressible hot fluid is considered at a depth $h$ in the $z$-axis with coordinate $(0, h)$ shown in Figure 1. 


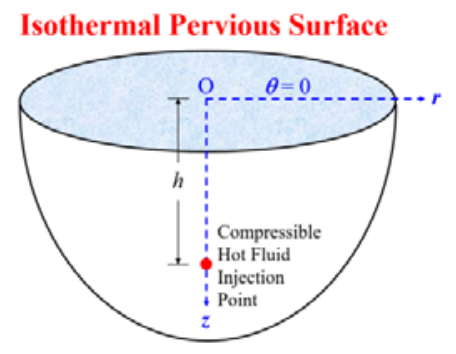

(A)

Adiabatic Impervious Surface

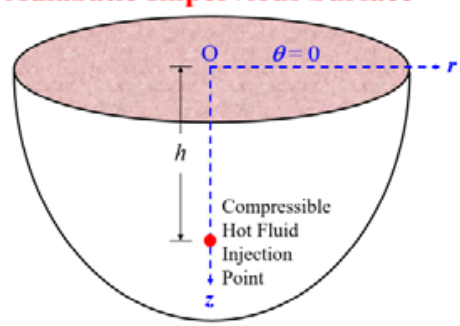

(B)

FIGURE I. COMPRESSIBLE HOT FLUID INJECTION INTO A POROUS ELASTIC HALF SPACE: (A) ISOTHERMAL PERVIOUS SURFACE. (B)ADIABATIC IMPERVIOUS SURFACE.

Using the laws of mass balance, energy conservation, continuity equation and heat conduction equation, the governing equations for fluid flow and thermal flow are derived in equations (1c) and (1d). With effect of body forces neglected, the axisymmetric governing equations are expressed in terms of radial horizontal displacement $u_{r}$, vertical displacement $u_{z}$, excess pore fluid pressure $p$, and temperature increment $\vartheta$ of the strata in axially symmetric cylindrical coordinates $(r, z)$ with the z-axis through the injection point as follows:

$$
\begin{gathered}
2 \eta\left(\frac{\partial^{2} u_{r}}{\partial r^{2}}+\frac{1}{r} \frac{\partial u_{r}}{\partial r}-\frac{u_{r}}{r^{2}}\right)+\frac{\partial^{2} u_{r}}{\partial z^{2}}+(2 \eta-1) \frac{\partial^{2} u_{z}}{\partial r \partial z} \\
-2(3 \eta-2) \alpha_{s} \frac{\partial \vartheta}{\partial r}-\frac{\alpha}{G} \frac{\partial p}{\partial r}=0, \\
(2 \eta-1)\left(\frac{\partial^{2} u_{r}}{\partial r \partial z}+\frac{1}{r} \frac{\partial u_{r}}{\partial z}\right)+\frac{\partial^{2} u_{z}}{\partial r^{2}}+\frac{1}{r} \frac{\partial u_{z}}{\partial r}+2 \eta \frac{\partial^{2} u_{z}}{\partial z^{2}} \\
-2(3 \eta-2) \alpha_{s} \frac{\partial \vartheta}{\partial z}-\frac{\alpha}{G} \frac{\partial p}{\partial z}=0, \\
\frac{k}{\gamma_{f}}\left(\frac{\partial^{2} p}{\partial r^{2}}+\frac{1}{r} \frac{\partial p}{\partial r}+\frac{\partial^{2} p}{\partial z^{2}}\right)+\frac{Q_{f}}{2 \pi r} \delta(r) \delta(z-h)=0,
\end{gathered}
$$

$$
\lambda_{t}\left(\frac{\partial^{2} \vartheta}{\partial r^{2}}+\frac{1}{r} \frac{\partial \vartheta}{\partial r}+\frac{\partial^{2} \vartheta}{\partial z^{2}}\right)+\frac{Q_{t}}{2 \pi r} \delta(r) \delta(z-h)=0 .
$$

The parameter $\eta=(1-v) /(1-2 v)$, and $v$ is Poisson's ratio of the strata. The shear modulus is denoted by $G$, and $\alpha_{s}$ represents the linear thermal expansion coefficient of the half space. The permeability and thermal conductivity of the half space are expressed as $k$ and $\lambda_{t}$, respectively. The parameter $\gamma_{f}$ is unit weight of injected fluid, and the symbols $\delta(r)$ and $\delta(z)$ are the Dirac delta functions. The thermal strength $Q_{t}$ of injected hot fluid is considered at a constant rate corresponding with fluid volume $Q_{f}$ per unit time. The compressible coefficient of pore fluid $\alpha$ is define by:

$$
\alpha=\frac{3\left(v_{u}-v\right)}{B(1-2 v)\left(1+v_{u}\right)},
$$

in which the Skempton pore fluid pressure coefficient $B$ and undrained Poisson's ratio $v_{u}$ were defined by Rice and Cleary [7].

\section{B. Boundary Conditions}

The half space ground surface at $z=0$ is treated as a traction-free boundary at time $t \geq 0$. Therefore, its analytical statements of the mechanical boundary conditions are:

$$
\sigma_{r z}(r, 0)=0, \sigma_{z z}(r, 0)=0
$$

where $\sigma_{i j}$ is the total stress components. The analytical statements of the isothermal pervious and adiabatic impervious conditions at the boundary $z=0$ are given as the following equation (4a) and (4b), respectively.

Case A. Isothermal pervious half space,

$$
p(r, 0)=0, \vartheta(r, 0)=0 .
$$

Case B. Adiabatic impervious half space,

$$
\frac{\partial p(r, 0)}{\partial z}=0, \frac{\partial \vartheta(r, 0)}{\partial z}=0 .
$$

The above two cases of hot fluid injection are assumed with no effect at the remote boundary of $z \rightarrow \infty$ at time $t \geq 0$. Hence,

$$
\lim _{z \rightarrow \infty}\left\{u_{r}(r, z), u_{z}(r, z), p(r, z), \vartheta(r, z)\right\}=\{0,0,0,0\} .
$$


The responses can be derived from differential equations (1a), (1b), (1c), and (1d) corresponding with half space boundary conditions (3), (4a) or (4b), and remote boundary condition (5).

\section{CLOSED-FORM SOLUTIONS}

Applying Hankel transform [8-10] with respect to the radial coordinate variable $r$ to the analytical model, the closed-form solutions in the transformed domain $(z ; \xi)$ are determined as below.

Case A. Isothermal pervious half space,

$$
\begin{aligned}
& \tilde{u}_{r}(z ; \xi)=\left[\frac{Q_{f} \gamma_{f}}{16 \pi G \eta k}+\frac{(3 \eta-2) \alpha_{s} Q_{t}}{8 \pi \eta \lambda_{t}}\right]\left\{\left(\frac{|z-h|}{\xi}+\frac{1}{\xi^{2}}\right) e^{-\xi|z-h|}\right. \\
& \left.+\left(-\frac{1}{\xi^{2}}+\frac{2 \eta+1}{2 \eta-1} \frac{h}{\xi}-\frac{z}{\xi}-2 h z\right) e^{-\xi(z+h)}\right\} \text {, } \\
& \tilde{u}_{z}(z ; \xi)=\left[\frac{Q_{f} \gamma_{f}}{16 \pi G \eta k}+\frac{(3 \eta-2) \alpha_{s} Q_{t}}{8 \pi \eta \lambda_{t}}\right]\left\{\frac{z-h}{\xi} e^{-\xi|z-h|}\right. \\
& \left.+\left(-\frac{2 \eta+1}{2 \eta-1} \frac{h}{\xi}-\frac{z}{\xi}-2 h z\right) e^{-\xi(z+h)}\right\} \text {, } \\
& \tilde{p}(z ; \xi)=\frac{\alpha Q_{f} \gamma_{f}}{4 \pi k} \frac{1}{\xi}\left\{e^{-\xi|z-h|}-e^{-\xi(z+h)}\right\}, \\
& \tilde{\vartheta}(z ; \xi)=\frac{Q_{t}}{4 \pi \lambda_{t}} \frac{1}{\xi}\left\{e^{-\xi|z-h|}-e^{-\xi(z+h)}\right\} .
\end{aligned}
$$

Case B. Adiabatic impervious half space,

$$
\begin{gathered}
\tilde{u}_{r}(z ; \xi)=\left[\frac{Q_{f} \gamma_{f}}{16 \pi G \eta k}+\frac{(3 \eta-2) \alpha_{s} Q_{t}}{8 \pi \eta \lambda_{t}}\right]\left\{\left(\frac{|z-h|}{\xi}+\frac{1}{\xi^{2}}\right) e^{-\xi|z-h|}\right. \\
\left.+\left(-\frac{1}{\xi^{2}}+\frac{2 \eta+1}{2 \eta-1} \frac{h}{\xi}+(4 \eta-1) \frac{z}{\xi}-2 h z\right) e^{-\xi(z+h)}\right\} \\
+\frac{(3 \eta-2) \alpha_{s} Q_{t}}{2 \pi \lambda_{t}}\left(\frac{1}{2 \eta-1} \frac{1}{\xi^{2}}-\frac{z}{\xi}\right) e^{-\xi(z+h)}, \\
\tilde{u}_{z}(z ; \xi)=\left[\frac{Q_{f} \gamma_{f}}{16 \pi G \eta k}+\frac{(3 \eta-2) \alpha_{s} Q_{t}}{8 \pi \eta \lambda_{t}}\right]\left\{\frac{z-h}{\xi} e^{-\xi|z-h|}\right. \\
\left.+\left[-\frac{2 \eta+1}{2 \eta-1} \frac{h}{\xi}+\frac{8 \eta^{2}}{2 \eta-1} \frac{1}{\xi^{2}}+(4 \eta-1) \frac{z}{\xi}-2 h z\right] e^{-\xi(z+h)}\right\}
\end{gathered}
$$

$$
\begin{gathered}
+\frac{(3 \eta-2) \alpha_{s} Q_{t}}{2 \pi \lambda_{t}}\left(-\frac{2 \eta+1}{2 \eta-1} \frac{1}{\xi^{2}}-\frac{z}{\xi}\right) e^{-\xi(z+h)} \\
-\frac{Q_{f} \gamma_{f}}{16 \pi G \eta k} \frac{4 \eta}{2 \eta-1} \frac{1}{\xi^{2}} e^{-\xi(z+h)}, \\
\tilde{p}(z ; \xi)=\frac{\alpha Q_{f} \gamma_{f}}{4 \pi k} \frac{1}{\xi}\left\{e^{-\xi|z-h|}+e^{-\xi(z+h)}\right\},
\end{gathered}
$$

$$
\tilde{\vartheta}(z ; \xi)=\frac{Q_{t}}{4 \pi \lambda_{t}} \frac{1}{\xi}\left\{e^{-\xi|z-h|}+e^{-\xi(z+h)}\right\}
$$

The symbol $\xi$ is Hankel transform parameter, and $\tilde{u}_{r}(z ; \xi)$, $\tilde{u}_{z}(z ; \xi), \tilde{p}(z ; \xi)$ and $\tilde{\vartheta}(z ; \xi)$ are defined as:

$$
\begin{aligned}
& \tilde{u}_{r}(z ; \xi)=\int_{0}^{\infty} r u_{r}(r, z) J_{0}(\xi r) d r, \\
& \tilde{u}_{z}(z ; \xi)=\int_{0}^{\infty} r u_{z}(r, z) J_{1}(\xi r) d r, \\
& \tilde{p}(z ; \xi)=\int_{0}^{\infty} r p(r, z) J_{0}(\xi r) d r, \\
& \tilde{\vartheta}(z ; \xi)=\int_{0}^{\infty} r \vartheta(r, z) J_{0}(\xi r) d r,
\end{aligned}
$$

in which $J_{n}(\xi r)$ represents the first kind of Bessel function of order $n$. The Hankel inversions of equations (8a) to (8d) are:

$$
\begin{aligned}
& u_{r}(r, z)=\int_{0}^{\infty} \xi \tilde{u}_{r}(z ; \xi) J_{0}(\xi r) d \xi, \\
& u_{z}(r, z)=\int_{0}^{\infty} \xi \tilde{u}_{z}(z ; \xi) J_{1}(\xi r) d \xi, \\
& p(r, z)=\int_{0}^{\infty} \xi \tilde{p}(z ; \xi) J_{0}(\xi r) d \xi, \\
& \vartheta(r, z)=\int_{0}^{\infty} \xi \tilde{\vartheta}(z ; \xi) J_{0}(\xi r) d \xi .
\end{aligned}
$$

Applying the Hankel inversions to formulae (6a) to (6d) and (7a) to (7d), the closed-form solutions of radial horizontal 
displacement $u_{r}$, vertical displacement $u_{z}$, excess pore fluid pressure $p$, and temperature increment $\vartheta$ of the strata due to a point of hot fluid injection into an isotropic half space are obtained as below.

Case A. Isothermal pervious half space,

$$
\begin{gathered}
u_{r}(r, z)=\frac{1}{16 \pi \eta}\left[\frac{Q_{f} \gamma_{f}}{k G}+\frac{2(3 \eta-2) \alpha_{s} Q_{t}}{\lambda_{t}}\right] \\
\cdot\left[\frac{r}{R_{a}}-\frac{r}{R_{b}^{*}}+\frac{2 \eta+1}{2 \eta-1} \frac{r h}{R_{b} R_{b}^{*}}-\frac{r z}{R_{b} R_{b}^{*}}-\frac{2 h r z}{R_{b}^{3}}\right], \\
u_{z}(r, z)=\frac{1}{16 \pi \eta}\left[\frac{Q_{f} \gamma_{f}}{k G}+\frac{2(3 \eta-2) \alpha_{s} Q_{t}}{\lambda_{t}}\right] \\
\cdot\left[\frac{z-h}{R_{a}}-\frac{2 \eta+1}{2 \eta-1} \frac{h}{R_{b}}-\frac{z}{R_{b}}-\frac{2 h z(z+h)}{R_{b}^{3}}\right], \\
p(r, z)=\frac{\alpha Q_{f} \gamma_{f}}{4 \pi k}\left(\frac{1}{R_{a}}-\frac{1}{R_{b}}\right), \\
\vartheta(r, z)=\frac{Q_{t}}{4 \pi \lambda_{t}}\left(\frac{1}{R_{a}}-\frac{1}{R_{b}}\right) .
\end{gathered}
$$

Case B. Adiabatic impervious half space,

$$
\begin{gathered}
u_{r}(r, z)=\frac{Q_{f} \gamma_{f}}{16 \pi G \eta k}\left[\frac{r}{R_{a}}-\frac{r}{R_{b}^{*}}\right. \\
\left.+\frac{2 \eta+1}{2 \eta-1} \frac{r h}{R_{b} R_{b}^{*}}+(4 \eta-1) \frac{r z}{R_{b} R_{b}^{*}}-\frac{2 h r z}{R_{b}^{3}}\right] \\
+\frac{(3 \eta-2) \alpha_{s} Q_{t}}{8 \pi \eta \lambda_{t}}\left[\frac{r}{R_{a}}+\frac{2 \eta+1}{2 \eta-1} \frac{r}{R_{b}^{*}}\right. \\
\left.+\frac{2 \eta+1}{2 \eta-1} \frac{h r}{R_{b} R_{b}^{*}}-\frac{r z}{R_{b} R_{b}^{*}}-\frac{2 h r z}{R_{b}^{3}}\right], \\
u_{z}(r, z)=\frac{Q_{f} \gamma_{f}}{16 \pi G \eta k}\left[\frac{z-h}{R_{a}}-\frac{2 \eta+1}{2 \eta-1} \frac{h}{R_{b}}+(4 \eta-1) \frac{z}{R_{b}}\right. \\
\left.-\frac{2 h z(z+h)}{R_{b}^{3}}-4 \eta \sinh ^{-1} \frac{z+h}{r}\right] \\
+\frac{(3 \eta-2) \alpha_{s} Q_{t}}{8 \pi \eta \lambda_{t}}\left[\frac{z-h}{R_{a}}-\frac{2 \eta+1}{2 \eta-1} \frac{h}{R_{b}^{*}}-\frac{z}{R_{b}}\right.
\end{gathered}
$$

$$
\begin{gathered}
\left.-\frac{2 h z(z+h)}{R_{b}^{3}}+\frac{4 \eta}{2 \eta-1} \sinh ^{-1} \frac{z+h}{r}\right], \\
p(r, z)=\frac{\alpha Q_{f} \gamma_{f}}{4 \pi k}\left(\frac{1}{R_{a}}+\frac{1}{R_{b}}\right), \\
\vartheta(r, z)=\frac{Q_{t}}{4 \pi \lambda_{t}}\left(\frac{1}{R_{a}}+\frac{1}{R_{b}}\right) .
\end{gathered}
$$

The distance symbols $R_{a}, R_{b}, R_{a}^{*}$ and $R_{b}^{*}$ in equations (10a) to (10d) and (11a) to (11d) are defined in equations (12a) to (12d):

$$
\begin{gathered}
R_{a}=\sqrt{r^{2}+(z-h)}, \\
R_{b}=\sqrt{r^{2}+(z+h)^{2}}, \\
R_{a}^{*}=\sqrt{r^{2}+(z-h)^{2}}+|z-h|, \\
R_{b}^{*}=\sqrt{r^{2}+(z+h)^{2}}+z+h .
\end{gathered}
$$

The long-term displacement of the closed-form solutions (10a), (10b), (11a) and (11b) contain two parameters $Q_{t}$ and $Q_{f}$; $Q_{t}$ is the constant thermal strength, and $Q_{f}$ describes the fluid volume effect per unit time under the injection of hot fluid into a porous elastic half space. All of the derived field quantities are functions of the distance from the hot fluid injection source, and these quantities are inversely proportional to the hydraulic permeability or thermal conductivity. Besides, the mechanical moduli shear modulus $G$ and Poisson's ratio $v$ do not have influence on the long-term excess pore fluid and temperature increment of the strata.

\section{CONCLUSIONS}

Based on the theory of thermal elasticity of porous media, the long-term closed-form solutions of a homogeneous isotropic elastic half space for axially symmetric deformations, excess pore fluid pressure, and temperature increment subjected to a point injection of compressible hot fluid are presented by equations (10a) to (10d) and (11a) to (11d). The displacement expressions of the closed-form solutions contains one parameter for the constant thermal strength $Q_{t}$ and another parameter describing the fluid volume $Q_{f}$ effect per unit time under the injection of hot fluid into the porous elastic strata. Besides, the 
long-term behavior of excess pore fluid pressure of the strata is affected by fluid volume $Q_{f}$, while the thermal strength $Q_{t}$ has an influence on the long-term temperature increment of the half space. The results can improve understanding of the hot fluid injection induced responses of homogeneous isotropic porous elastic half space.

\section{ACKNOWLEDGMENT}

This work is supported by the Ministry of Science and Technology of Taiwan, Republic of China, through grants NSC90-2211-E-216-020 and MOST106-2625-M-415-003.

\section{REFERENCES}

[1] J. Bear, and M. Y. Corapcioglu, "A mathematical model for consolidation in a thermoelastic aquifer due to hot water injection or pumping," Water Resources Research, vol. 17, no. 3, pp. 723-736, 1981.

[2] A. F. Alajmi, R. Gharbi, and M. Algharaib, "Investigating the performance of hot water injection in geostatistically generated permeable media,” Journal of Petroleum Science and Engineering, vol. 66, no. 3-4, pp. 143-155, 2009.

[3] K. Sasaki, S. Ono, Y. Sugai, T. Ebinuma, H. Narita, and T. Ya-maguchi, "Gas production system from methane hydrate layers by hot water injection using dual horizontal wells,” Journal of Canadian Petroleum Technology, vol. 48, no. 10, pp. 21-26, 2009.

[4] E. Rosenbrand, C. Haugwitz, P. S. M. Jacobsen, C. Kjoller, and I. L. Fabricius, "The effect of hot water injection on sandstone permeability," Geothermics, vol. 50, pp. 155-166, 2014.

[5] J. C.-C. Lu, and F.-T. Lin, "The transient ground surface displacements due to a point sink/heat source in an elastic half-space," Geotechnical Special Publication, no. 148, pp. 210-218, 2006.

[6] X. Yang, C.-Y. Sun, Q. Yuan, P.-C. Ma, and G.-J Chen, "Experimental study on gas production from methanehy-drate-bearing sand by hot-water cyclic injection,” Energy Fuels, vol. 24, no. 11, pp. 5912-5920, 2010.

[7] J. R. Rice, and M. P. Cleary, "Some basic stress diffusion solutions for fluid-saturated elastic porous media with compressible constitutents," Reviews of Geophysics and Space Physics, vol. 14, no. 2, pp. 227-241, 1976.

[8] I. N. Sneddon, Fourier Transforms, McGraw-Hill, New York, 1951.

[9] I. S. Gradshteyn, and I. M. Ryzhik, Table of Integrals, Series, and Products, Academic Press, New York, 1980.

[10] A. Erdelyi, W. Magnus, F. Oberhettinger, and F. G. Tricomi, Tables of Integral Transforms, McGraw-Hill, New York, 1954.

\section{NOTATION OF SYMBOLS}

$B$ Skempton pore fluid pressure coefficient of the isotropic strata (Dimensionless)

$G \quad$ Shear modulus of the isotropic strata $(\mathrm{Pa})$

$h \quad$ Depth of compressible hot fluid injection point (m)

$J_{n}(x)$ The first kind of Bessel function of order $n$ (Dimensionless)

$k \quad$ Permeability of the isotropic strata $(\mathrm{m} / \mathrm{s})$

$p \quad$ Excess pore fluid pressure of the isotropic strata $(\mathrm{Pa})$

$\tilde{p} \quad$ Hankel transform of $p(\mathrm{~N})$

$Q_{f} \quad$ Fluid volume of the injected hot fluid per unit time $\left(\mathrm{m}^{3} / \mathrm{s}\right)$

$Q_{t} \quad$ Thermal strength of the injected hot fluid (J/s) $(r, \theta, z)$ Cylindrical coordinates system (m, radian, $\mathrm{m})$

$R_{a} \quad$ Distance parameter defined in equation (12a) (m)

$R_{a}^{*} \quad$ Distance parameter defined in equation (12c) (m)

$R_{b} \quad$ Distance parameter defined in equation (12b) (m)

$R_{b}^{*} \quad$ Distance parameter defined in equation (12d) (m)

$t \quad$ Time variable (s)

$u_{r} \quad$ Radial horizontal displacement of the isotropic strata (m)

$\tilde{u}_{r} \quad$ Hankel transform of $u_{r}\left(\mathrm{~m}^{3}\right)$

$u_{z} \quad$ Vertical displacement of the isotropic strata (m)

$\tilde{u}_{z} \quad$ Hankel transform of $u_{z}\left(\mathrm{~m}^{3}\right)$

$\alpha \quad$ Compressible coefficient of pore fluid (Dimensionless)

$\alpha_{s} \quad$ Linear thermal expansion coefficient of the isotropic strata $\left({ }^{\circ} \mathrm{C}^{-1}\right)$

$\gamma_{f} \quad$ Unit weight of injected fluid $\left(\mathrm{N} / \mathrm{m}^{3}\right)$

$\delta(x) \quad$ Dirac delta functions $\left(\mathrm{m}^{-1}\right)$

$\eta \quad$ Parameter, $\eta=(1-v) /(1-2 v)$ (Dimensionless)

$\vartheta \quad$ Temperature changes of the isotropic strata $\left({ }^{\circ} \mathrm{C}\right)$

$\tilde{\vartheta} \quad$ Hankel transform of $\vartheta\left({ }^{\circ} \mathrm{C} \mathrm{m}^{2}\right)$

$\lambda_{t} \quad$ Thermal conductivity of the isotropic strata $\left(\mathrm{J} / \mathrm{sm}^{\circ} \mathrm{C}\right)$

$v \quad$ Poisson's ratio of the isotropic strata (Dimensionless)

$v_{u} \quad$ Undrained Poisson's ratio of the isotropic strata (Dimensionless)

$\xi \quad$ Hankel transform parameter $\left(\mathrm{m}^{-1}\right)$

$\sigma_{i j} \quad$ Total stress components of the isotropic strata (Pa) 